\title{
Synthesis of Colloidal Quantum Dot-Based Heterostructures for Photon Upconversion
}

\author{
Tory Welsch, Jill Cleveland, Eric Chen, Christopher Milleville, Kyle Lennon, \\ Jing Zhang, James Bork, Joshua Zide, Matthew Doty \\ Department of Materials Science and Engineering, University of Delaware, Newark, DE 19716 \\ WEIIERSTTYof
}

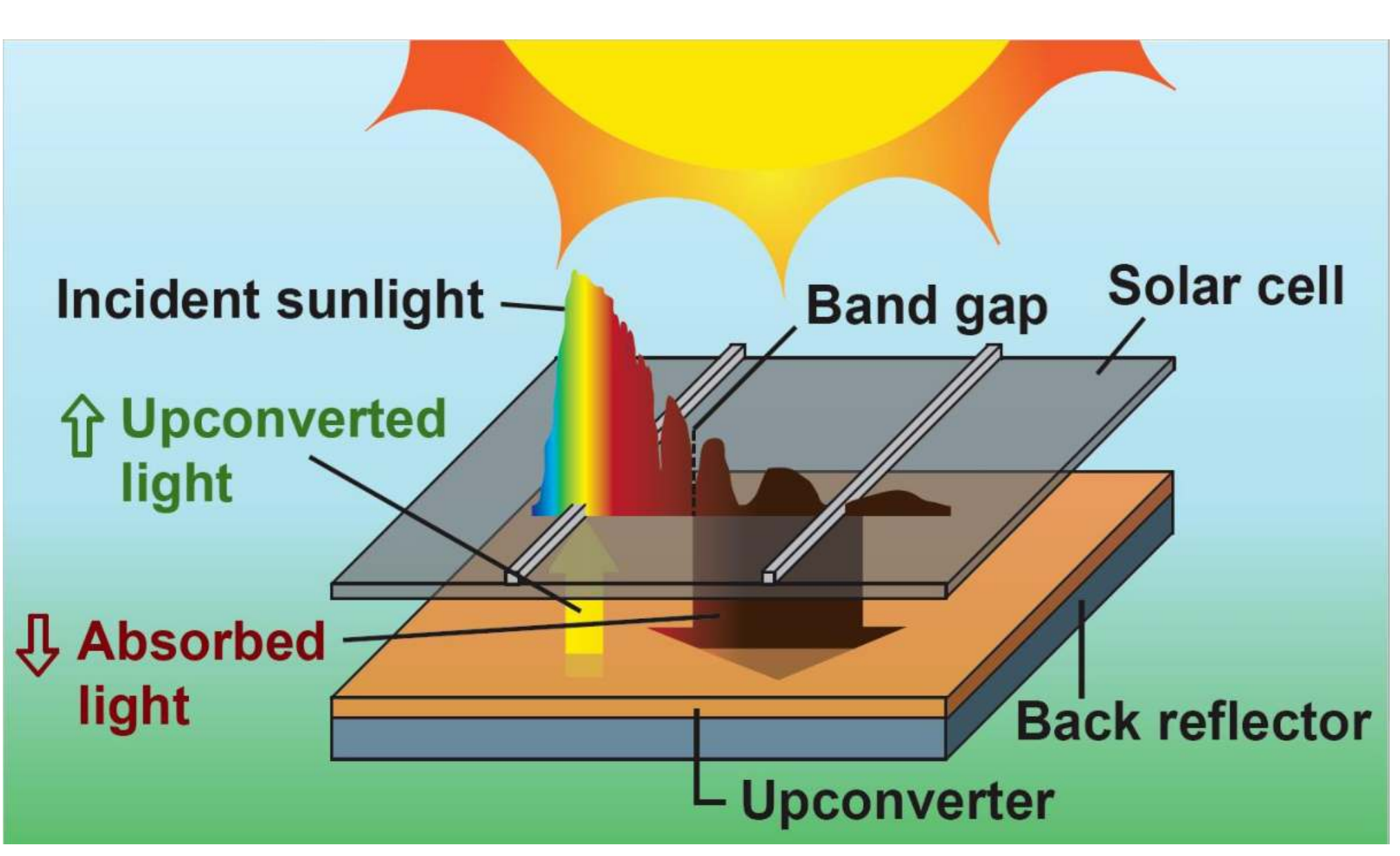

\section{What is photon upconversion?}

Upconversion is the process by which two low-energy photons are absorbed and re-emitted as a single higher-energy photon. An upconverter-backed solar cell can exceed the Shockley-Queisser limit by using upconverted sub-bandgap photons that otherwise cannot be absorbed by conventional solar cells.

\section{Why colloidal semiconductor nanostructures?}

Semiconductors offer unique advantages over other upconverting materials because they have a wide absorption bandwidth and their absorption and emission properties can be tuned by controlling their composition, shape, and size. These materials are also compatible with existing bulk processing methods used for solar cells.

\section{Recent Progress: Nanorod platform}

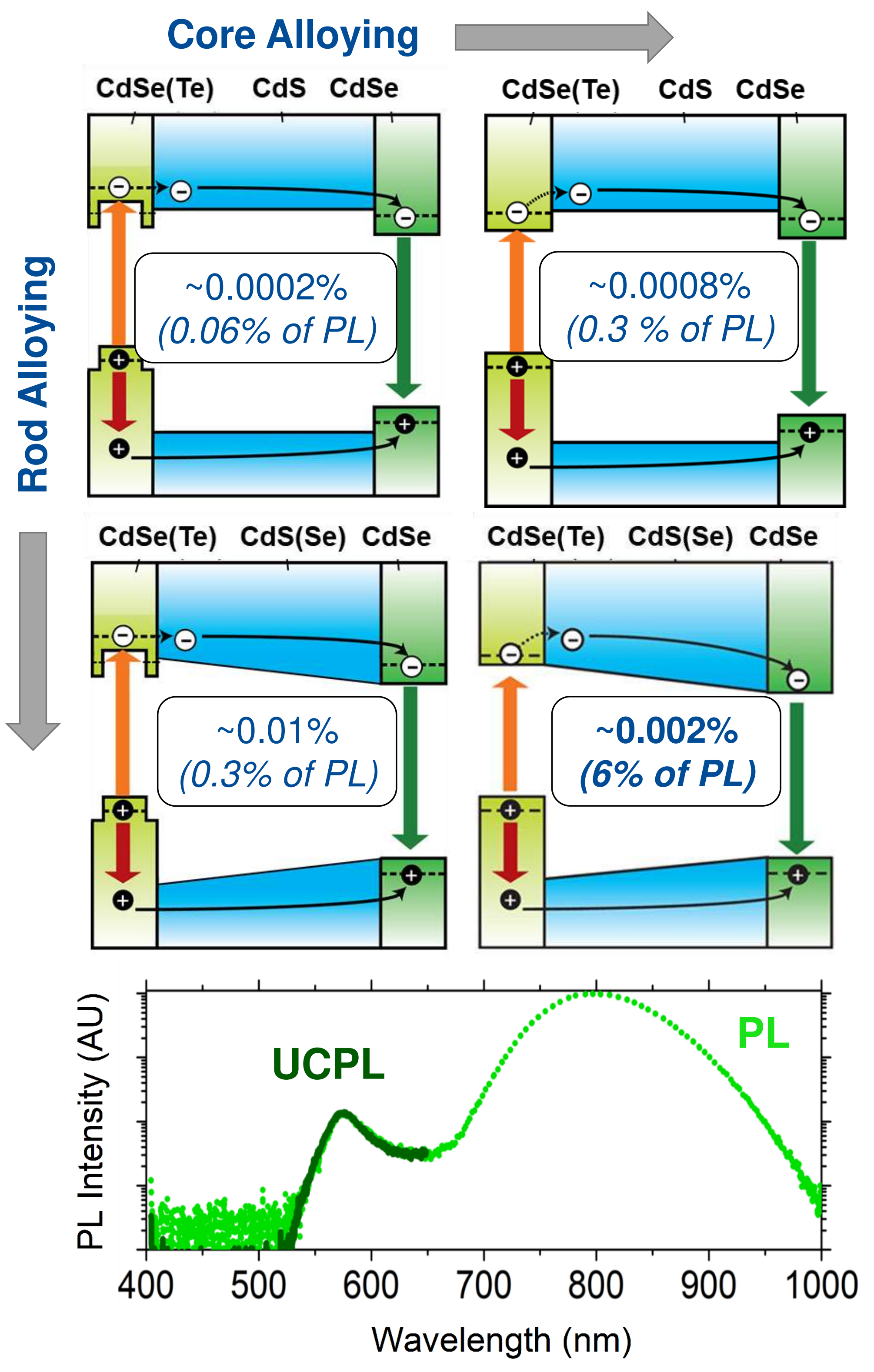

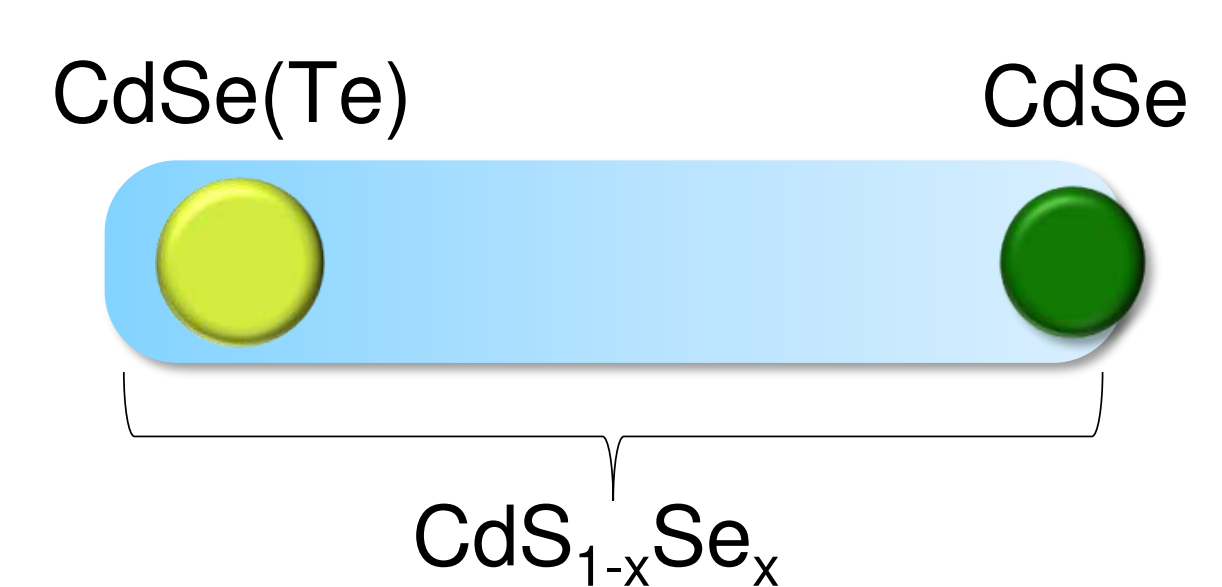

Weaknesses

- Unreliable synthesis methods limit structure quality

- Surface and interface defects result in low PLQY

- Core/rod structure restricts possible material types

\section{Strengths}

- NR important for sufficient charge carrier separation

- Alloying helps funnel carriers to the emitter

- 100x improvement in UCPL/PL ratio through heterostructure engineering
I. Synthesis of Ternary CdSe(Te) QDs

CdO:TDPA in ODE Se (90\%):Te (10\%):TOP Hot injection at $305^{\circ} \mathrm{C}$ Cd:Se/Te

\section{Seeded-Growth of $\mathrm{CdS}_{1-\mathrm{x}} \mathrm{Se}_{\mathrm{x}} \mathrm{NRs}$}

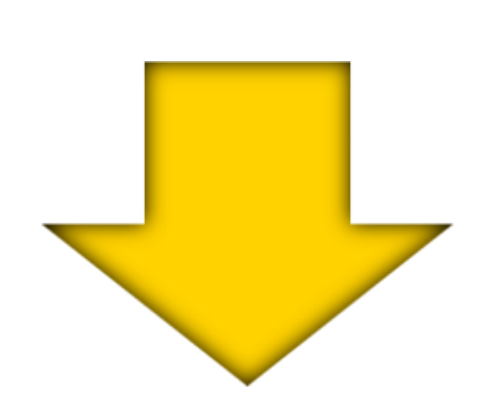

$$
\begin{aligned}
& \text { CdO:ODPA/HPA/TOPO } \\
& \text { S:TOP } \\
& \text { CdSe(Te) QDs }
\end{aligned}
$$

Hot injection at $350^{\circ} \mathrm{C}$

III. SILAR Growth of CdSe QDs

(Successive lonic Layer Adsorption and Reaction)

$$
\text { CdO:TOP }
$$

Se:TOP

CdSe(Te)@CdS(Se) NRs in TOPO $200^{\circ} \mathrm{C}$
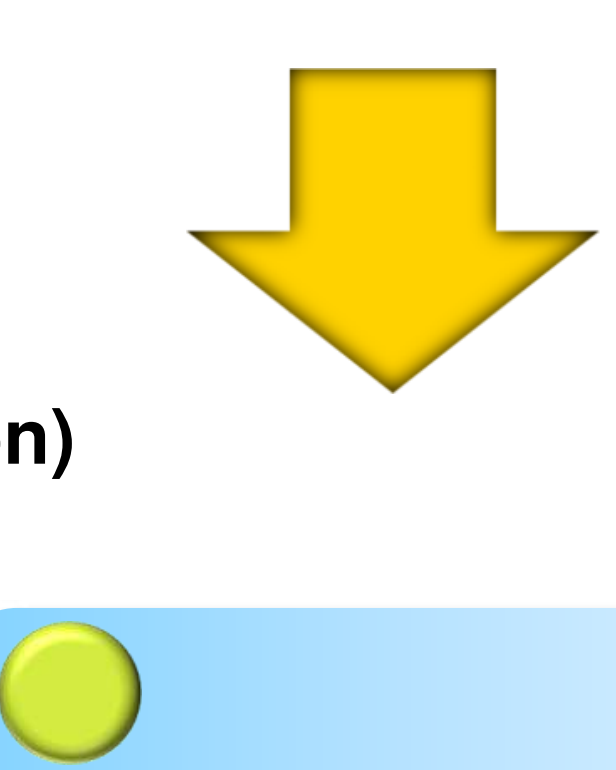

\section{Current Focus: Core/shell platform}

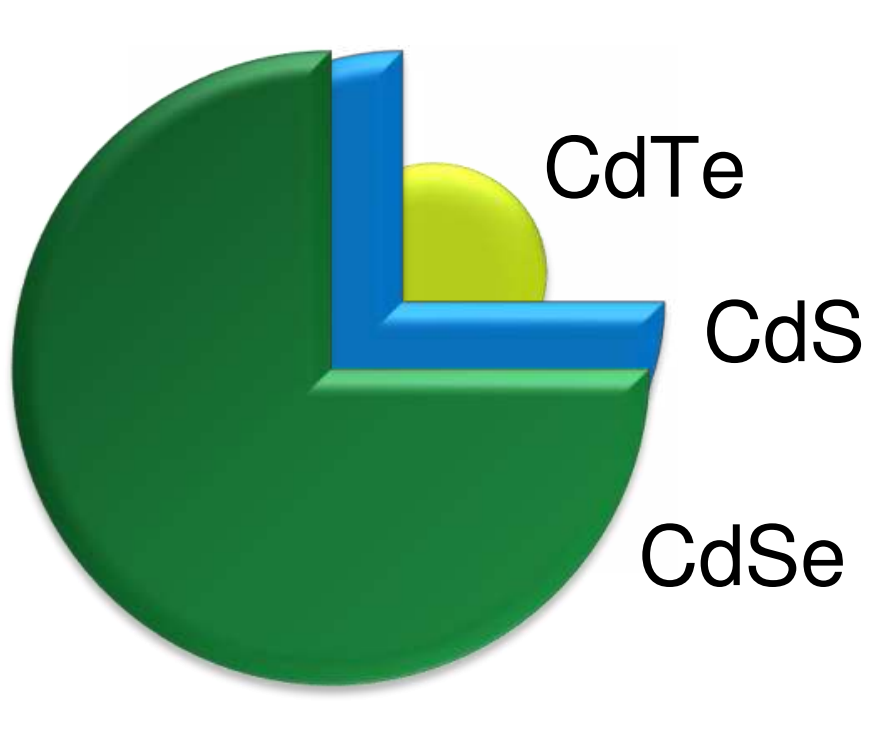

Strengths

- Ability to synthesize higher quality materials

- Addition of passivating shell can eliminate defects

- Core/shell platform compatible with many semiconductors

- Spacer layer thickness can be optimized bandgap gradient

- Opportunity to further improve upconversion performance
- Can introduce similar

\section{CdSe CdS CdTe CdS CdSe}
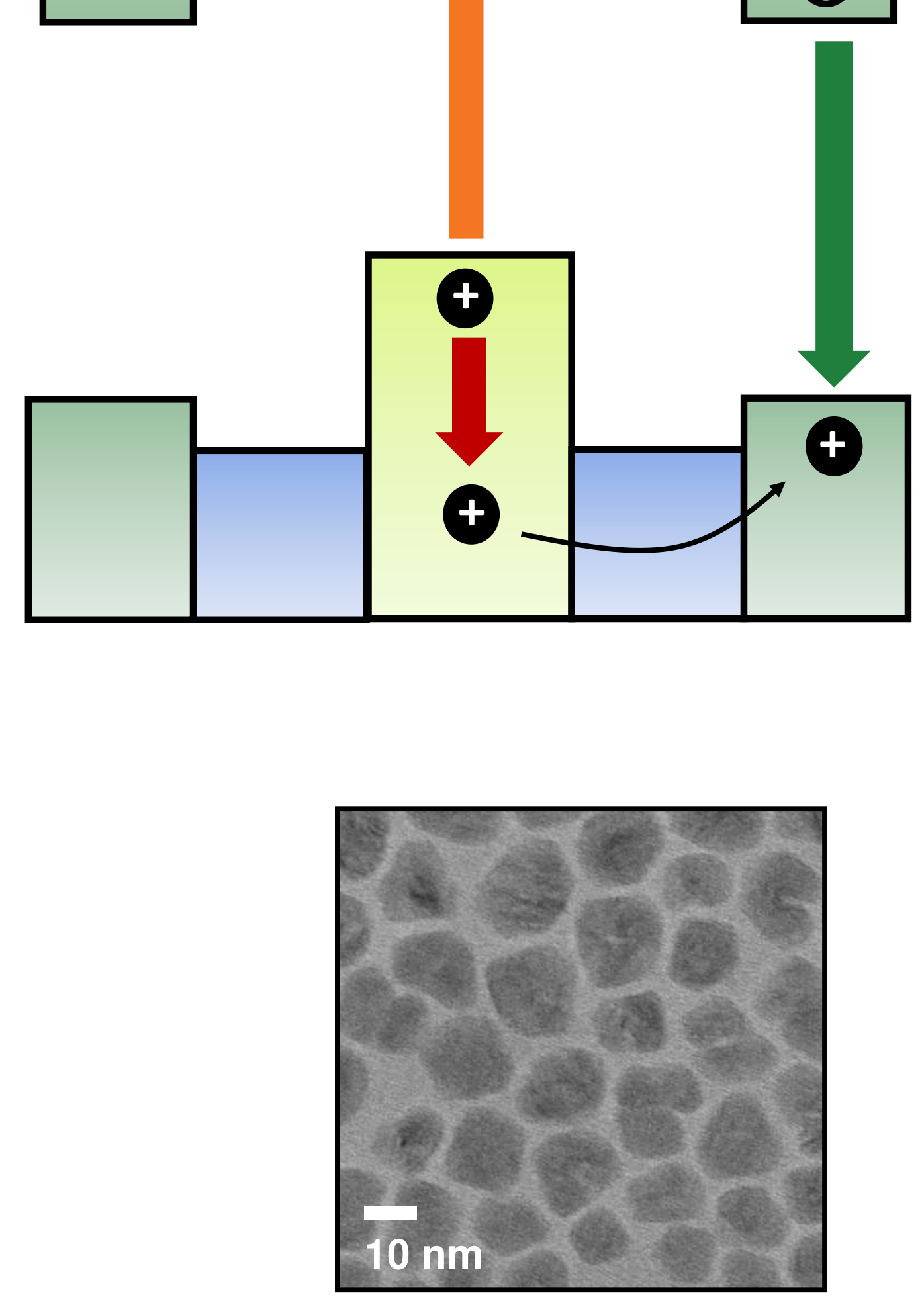

CdTe/CdS

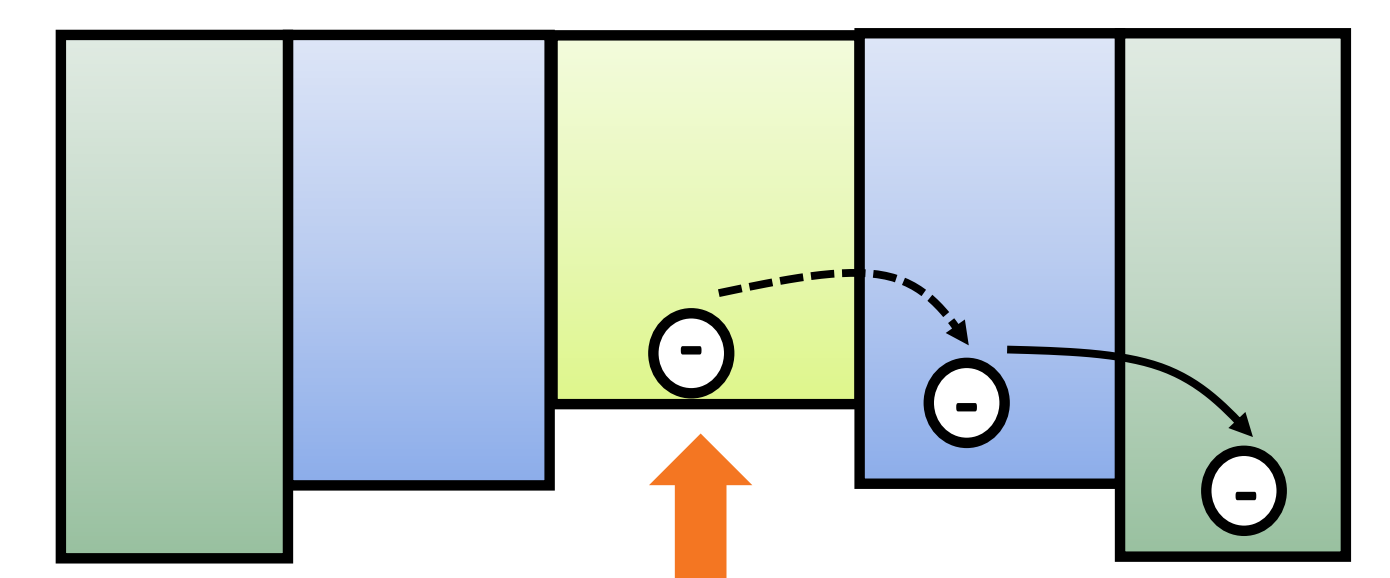

\section{Synthesis of CdTe QDs}

$\begin{array}{cc}\text { 1:10 } & \text { CdO:ODPA/TOPO } \\ \text { Cd:Te } & \text { Te:TOP } \\ & \text { Hot injection at } 320^{\circ} \mathrm{C}\end{array}$

\section{Addition of CdS Layer}

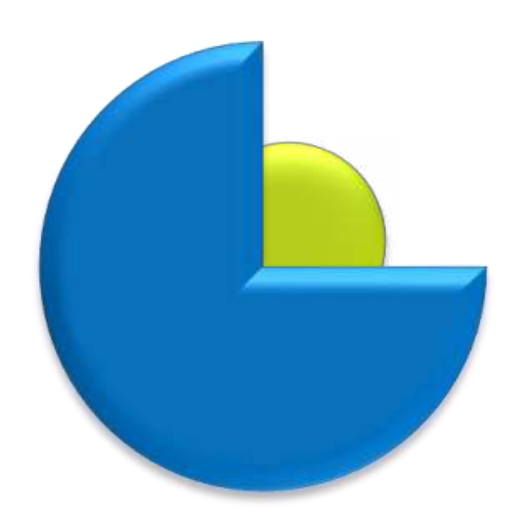

CdO:Oleic acid/TOPO

CdSe(Te)@CdS 1-x $S e_{x} @ C d S e$

(Successive lonic Layer Adsorption and Reaction)
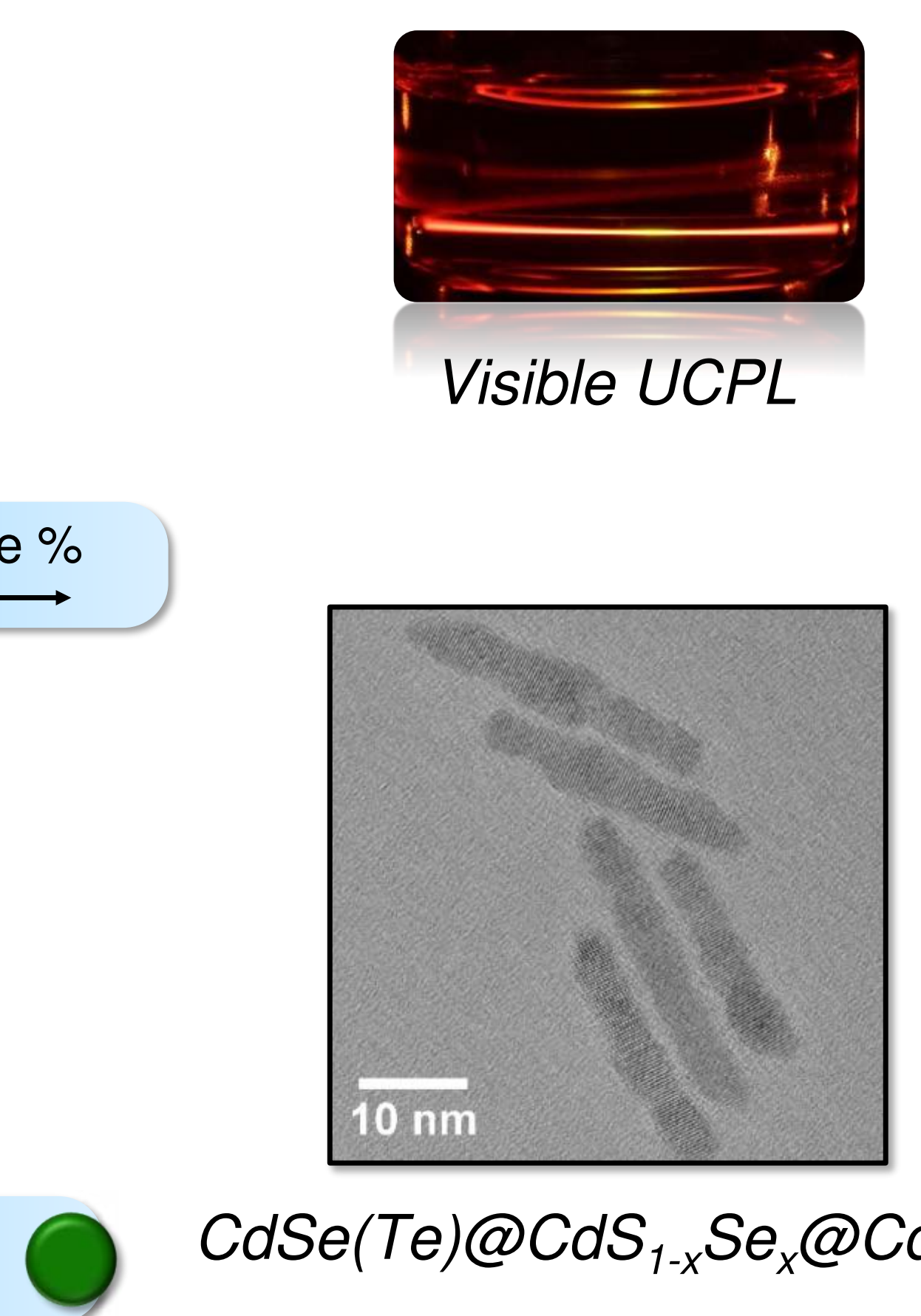

Visible UCPL
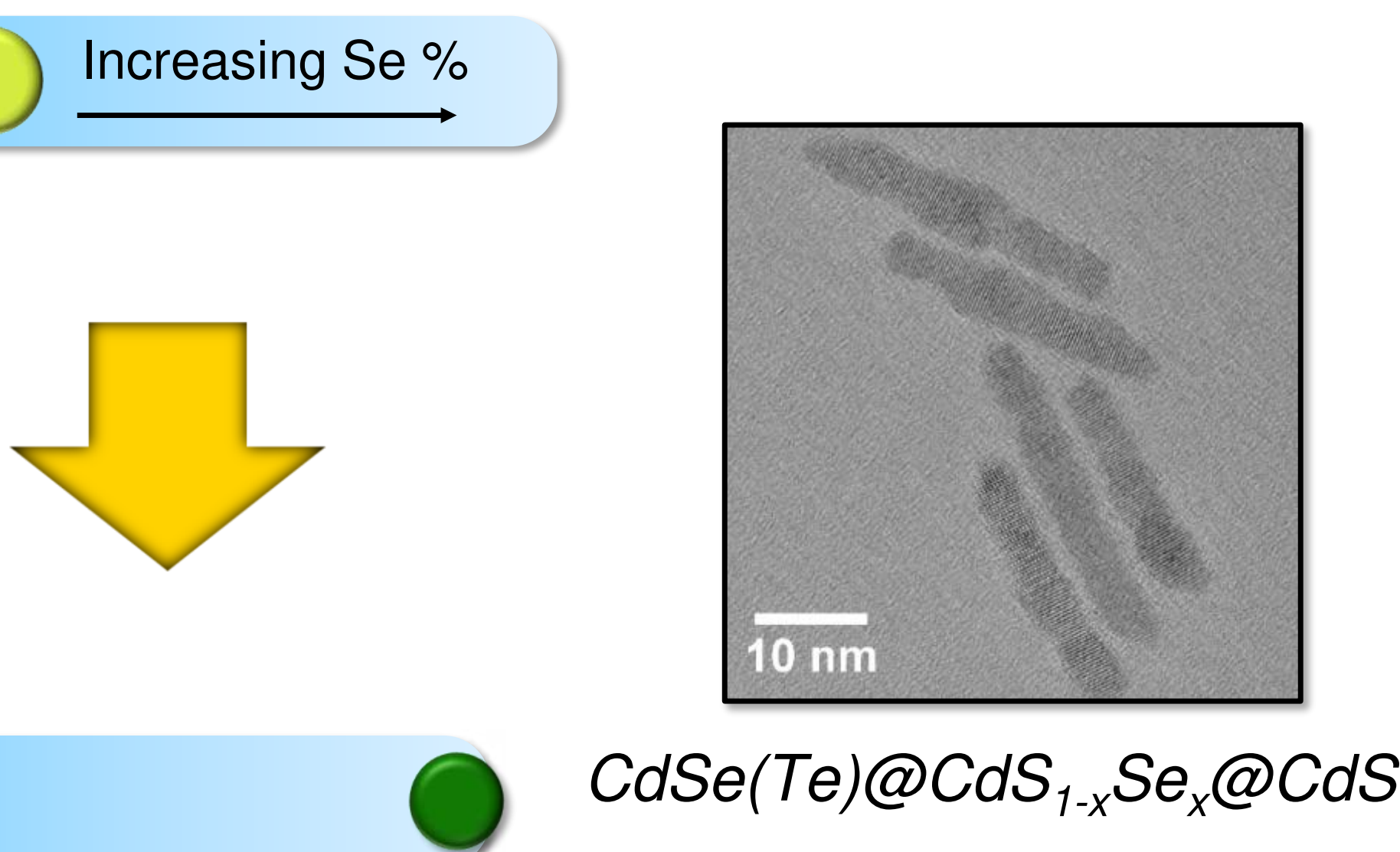

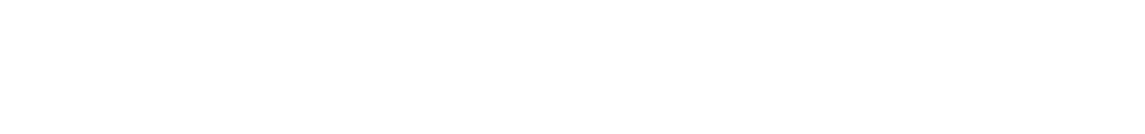

\section{References}

[1] Milleville, C. C. et al. ACS Nano 2019, 13 (1), 489-497.

\section{Acknowledgements}

III. SILAR Growth of CdSe Shell

CdO:Oleic acid/ODE

Se:TOP/ODE

$\mathrm{CdTe/CdS} \mathrm{core/shell} \mathrm{QDs} \mathrm{in}$

ODE/Oleylamine $150^{\circ} \mathrm{C}$ 